\title{
Aspectos clínicos de vasculitis retinianas secundarias
}

\section{Clinical features of secondary retinal vasculitis}

\author{
Irving Enrique Carral-Santander ${ }^{1 *}$, Luz Elena Concha del Rio ${ }^{2}$, Rashel Cheja-Kalb ${ }^{2}$ y \\ Lourdes Arellanes-García ${ }^{3}$
}

${ }^{1}$ Médico Oftalmólogo Actual Fellowship de Alta especialidad en Retina y Vítreo; ${ }^{2}$ Médico adscrito del servicio de enfermedades inflamatorias oculares; '3efe de Servicio del servicio de enfermedades inflamatorias oculares. Asociación Para Evitar la Ceguera en México, Hospital Dr. Luis Sánchez Bulnes, Ciudad de México, México

\section{Resumen}

Las vasculitis retinianas secundarias son manifestaciones oculares poco frecuentes de diversas enfermedades con componente autoinmune, como granulomatosis con poliangitis, enfermedad de Behcet, sarcoidosis, lupus eritematoso sistémico y esclerosis múltiple, entre otras. Debido a las repercusiones sistémicas el diagnóstico correcto es de mayor importancia. Los síntomas oftalmológicos más frecuentes son visión borrosa y miodesopsias. En algunos casos se observan signos oftalmológicos altamente sugestivos de ciertas enfermedades como la imagen de periflebitis retiniana en "gota de cera» de la sarcoidosis y el hipopion en la enfermedad de Behcet. Las manifestaciones sistémicas son esenciales para el diagnóstico por lo cual se deben reconocer las principales características de las enfermedades frecuentemente involucradas.

Palabras clave: Vasculitis retinianas secundarias. Sarcoidosis. Enfermedad de Behcet. Lupus eritematoso sistémico. Esclerosis múltiple.

\section{Abstract}

Secondary retinal vasculitis are unusual ocular manifestations of several autoimmune diseases, such as granulomatosis with polyangiitis, Behcet disease, sarcoidosis, systemic lupus erythematosus and multiple sclerosis, among others. As a consequence of systemic repercussions the correct diagnosis is of greater importance. The most frequent ophthalmological symptoms are blurry vision and floaters. In some cases highly suggestive ophthalmological signs of certain diseases might be presented, such as retinal periphlebitis also known as "candle wax drippings" image of sarcoidosis and the hypophion in Behcet disease. Systemic manifestations are essential for a correct diagnosis, therefore main features of the frequently involved diseases shall be identified.

Key words: Secondary retinal vasculitis. Sarcoidosis. Behcet disease. Systemic lupus erythematosus. Multiple sclerosis.

\section{Correspondencia:}

*Irving Enrique Carral-Santander

E-mail: dr.irvingcarral@gmail.com

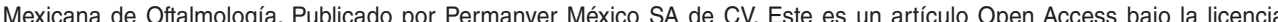
CC BY-NC-ND (http://creativecommons.org/licenses/by-nc-nd/4.0/).
Disponible en internet: 20-05-2016 Rev Mex Oftalmol. 2018;92(1):50-57 www.rmo.com.mx
Fecha de aceptación: 22-03-2016 DOI: 10.1016/j.mexoft.2016.03.009 
Tabla 1. Causas de vasculitis retinianas secundarias por etiología

\begin{tabular}{l|l|l|l|}
\hline Infecciosas & $\begin{array}{l}\text { Asociadas a enfermedades } \\
\text { neurológicas }\end{array}$ & Asociadas a neoplasia & Autoinmunes \\
\hline $\begin{array}{l}\text { Tuberculosis } \\
\text { Sífilis }\end{array}$ & $\begin{array}{l}\text { Esclerosis múltiple } \\
\text { Síndrome de Susac }\end{array}$ & $\begin{array}{l}\text { Paraneoplásicas } \\
\text { Linfoma ocular } \\
\text { Enfermedad de Lyme }\end{array}$ & $\begin{array}{l}\text { Sarcoidosis } \\
\text { Enfermedad } \\
\text { Enfermedad por arañazo de gato }\end{array}$ \\
$\begin{array}{l}\text { Toxoplasmosis } \\
\text { Citomegalovirus } \\
\text { Necrosis retiniana aguda }\end{array}$ & & \\
\hline
\end{tabular}

El término vasculitis de retina se refiere a la inflamación de arterias o venas retinianas de etiología autoinmune, infecciosa, neoplásica, medicamentosa o idiopática (Tabla 1). Se define como vasculitis primaria la vasculitis de retina donde existe una afección exclusiva de la vasculatura retiniana sin evidencia de enfermedad sistémica asociada; y como vasculitis secundaria la asociada a una enfermedad sistémica.

Las vasculitis secundarias pueden ocasionar visión borrosa y miodesopsias, ocasionalmente escotomas y algunos casos pueden ser asintomáticos. El signo oftalmológico característico que se va a encontrar es el envainamiento vascular (perivasculitis); la acumulación perivascular de material inflamatorio granulomatoso puede dar la imagen en "gotas de cera». La vasculitis puede ser oclusiva (Fig. 1), y en ella podemos observar datos de isquemia retiniana, hemorragias puntiformes y exudados algodonosos. Los cambios crónicos pueden ser cortocircuitos arteriovenosos, edema macular quístico o neovascularización coroidea.

En todos los casos deben descartarse etiologías infecciosas como tuberculosis, sífilis, y actualmente enfermedad de Lyme y «enfermedad por arañazo de gato». Una vez hecho esto se considerará la posibilidad de vasculitis de probable origen autoinmune, que como ya se dijo pueden ser primarias o secundarias.

Dentro del espectro de las vasculitis de retina secundarias se deben descartar las enfermedades que citamos a continuación.

\section{Lupus eritematoso sistémico}

Enfermedad autoinmune multisistémica, puede afectar cualquier órgano y tejido conectivo, cursa con remisiones y recaídas ${ }^{1}$.

\section{Epidemiología}

Es más común en africanos y asiáticos, las mayores complicaciones se dan en caucásicos, la máxima

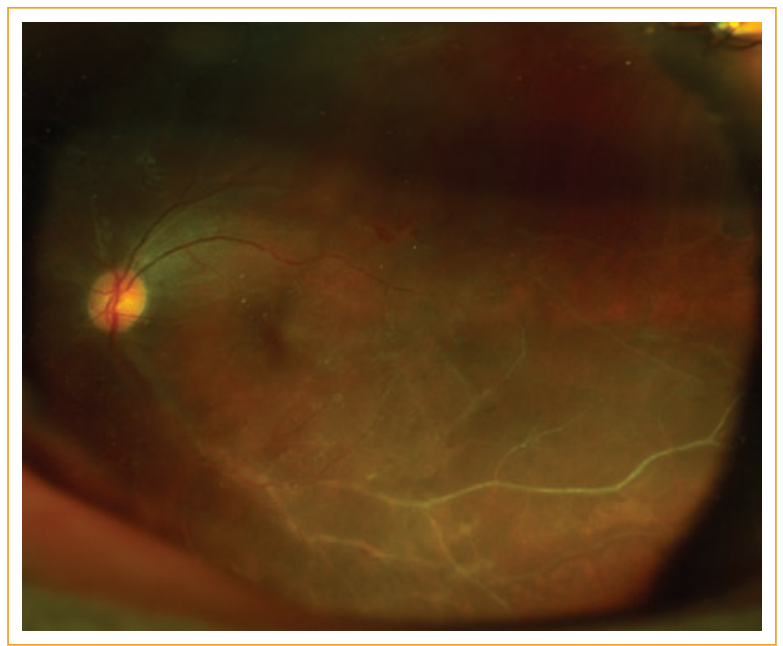

Figura 1. Fotografía de retina con vasculopatía oclusiva de la arcada temporal inferior.

prevalencia es en Italia, España y población del Reino Unido afro-caribe. La edad de presentación es desde la adolescencia tardía hasta los 40 años de edad, y 9 veces más común en mujeres. Asociación con HLA-A1, B8, DR3 ${ }^{1}$.

\section{Manifestaciones sistémicas}

Afección multisistémica muy variable. Dentro de estas se puede presentar artritis de tipo no erosivo y de predominio en articulaciones metacarpofalángicas e interfalángicas proximales, muñecas y rodillas; enfermedad mucocutánea: expresada en forma de rash malar, rash discoide, fotosensibilidad o úlceras orales habitualmente indoloras; alteraciones hematológicas: citopenias y/o trombofilia; alteraciones renales manifestadas por proteinuria y cilindros celulares; y afección de sistema nervioso central: disfunción cognitiva, síndrome cerebral orgánico, delirium, psicosis, crisis convulsivas, cefalea, corea y neuropatía periférica ${ }^{1-3}$. La tríada clásica de 
alteraciones hematológicas, enfermedad mucocutánea y rash malar en una mujer de edad reproductiva debe llevar a descartar este diagnóstico.

\section{Manifestaciones oculares}

Se presentan hasta en el $33 \%$ de los casos y la más frecuente es la queratoconjuntivitis sicca. Puede presentarse como alteración orbitaria, escleral y epiescleral, epiteliopatía corneal, queratitis ulcerativa periférica, coroidopatía o retinopatía lúpica (lesiones algodonosas, exudados duros perivasculares, hemorragias retinianas y tortuosidad vascular). En el $29 \%$ de los casos puede encontrarse vasculitis con exudados algodonosos con o sin hemorragia, la cual se considera señal de actividad de enfermedad. Otra forma es la vasculitis arteriolar oclusiva, que puede ser bilateral y se ha asociado con afección de sistema nervioso central. La isquemia resultante favorece la neovascularización, hemorragia vítrea y eventualmente desprendimiento traccional de retina ${ }^{4}$.

\section{Diagnóstico}

Para el diagnóstico de requieren 4 de los siguientes 11 criterios: rash malar, rash discoide, fotosensibilidad, úlceras orales, artritis no erosiva, serositis, disfunción renal, alteración neurológica como psicosis o crisis epilépticas, desórdenes hematológicos, desórdenes inmunológicos (anti-ADN, anti-S, falso positivo de VDRL) y presencia de anticuerpos antinucleares.

\section{Tratamiento}

Los corticoesteroides son la terapia más efectiva a corto plazo para el lupus eritematoso sistémico. Debido a la frecuente asociación de las alteraciones oculares con las del sistema nervioso central el uso de corticoesteroides intravenosos a altas dosis ha sido recomendado para prevenir la morbimortalidad. También se pueden usar esteroides perioculares en enfermedad unilateral o asimétrica. Debido a que el uso prolongado de corticoesteroides a altas dosis se asocia a un elevado número de complicaciones oculares como glaucoma (5$19 \%$ ) y catarata, los inmunosupresores se deben utilizar para la terapia a largo plazo; se ha reportado el uso de metrotexate, azatioprina, micofenolato de mofetilo, ciclosporina A y ciclofosfamida en bolos intravenosos. El pronóstico es malo para la función a largo plazo y la probabilidad de supervivencia varía en función al grado de afección orgánica sobre todo renal y del sistema nervioso central. Se puede agregar anticoagulantes y ácido acetilsalicílico cuando hay anticuerpos antifosfolípidos positivos por el mayor riesgo de vasculopatía oclusiva y también se utilizan para estabilizar la enfermedad retiniana para prevenir eventos vasculares ${ }^{5}$. Se ha descrito el uso de antipalúdicos como cloroquina o hidroxicloroquina ya que son muy eficaces en la reducción de reactivaciones futuras con menos efectos secundarios que los agentes alquilantes, sin embargo se conoce bien el daño macular que puede ocurrir por el uso progresivo de estos medicamentos. Existen reportes donde se describe el uso de agentes biológicos como rituximab en el manejo de estos pacientes ${ }^{1}$. Los pacientes con lupus no deben ser operados de cirugía refractiva ya que existe el riesgo de ectasia posquirúrgica, hecho que ha sido demostrado al estudiar los cambios en las propiedades biomecánicas de la córnea ${ }^{6}$.

\section{Esclerosis múltiple}

Enfermedad desmielinizante del sistema nervioso central crónica caracterizada por episodios de neuritis óptica, alteración en los movimientos oculares y múltiples síntomas neurológicos sistémicos asociados. Afecta varios aspectos del ojo.

\section{Epidemiología}

Afecta a adultos jóvenes entre 20 y 40 años de edad con predominio en mujeres: hombres en un radio de $3.5: 1$ y prevalencia de 1.5 por 100,000 habitantes en población mexicana ${ }^{7}$. Se ha descrito que el $1 \%$ de los casos pueden desarrollar inflamación intraocular ${ }^{8}$.

\section{Manifestaciones sistémicas}

Son causadas por el proceso desmielinizante, los síntomas varían debido a que la localización e intensidad de cada ataque puede ser diferente. Se han descrito parestesias, espasticidad, disfunción sexual, urinaria e intestinal. También se has descrito neuralgia del trigémino, mioquimia facial, síntomas constitucionales, depresión y mielitis transversa. Existe una asociación bien establecida entre el aumento de la temperatura corporal, debido a factores intrínsecos o extrínsecos, y un aumento de intensidad de los síntomas, esta se conoce como fenómeno de Uhthoff ${ }^{9}$.

\section{Manifestaciones oculares}

El $50 \%$ de los pacientes con esclerosis múltiple (EM) e inflamación ocular presentan neuritis óptica ${ }^{10}$, el tipo de 
uveítis más frecuentemente reportada es la intermedia, descrita hasta en el $78 \%$ de los casos: en el $12.5 \%$ de los ojos afectados puede observarse vasculitis, con afección de predominio venoso y en el $10 \%$ solo uveítis anterior ${ }^{11}$. Se ha reportado que el $15 \%$ de las uveítis intermedias se asocian a $\mathrm{EM}^{10}$. También se han descrito oftalmoplejía internuclear y nistagmo. Las complicaciones asociadas a la vasculitis son: obstrucciones vasculares, neovascularización de la retina y hemorragia vítrea. En la fluorangiografía de retina se puede encontrar periflebitis, zonas de isquemia retiniana focales por no perfusión de arteriolas retinianas y paredes arteriolares hiperfluorescentes ${ }^{12}$.

\section{Diagnóstico}

El diagnóstico es clínico, se confirma con la presencia de áreas de desmielinización periventriculares observadas en la resonancia magnética (T2/gadolinio). Se utilizan los criterios de McDonald para el diagnóstico'13 (Tabla 2).

\section{Tratamiento}

Una alternativa terapéutica son los bolos intravenosos de esteroides para episodios agudos de la enfermedad. El interferón clásicamente utilizado para EM con afección de sistema nervioso central, también se ha descrito en los últimos años para afección ocular, sin embargo faltan estudios que comprueben su efectividad ${ }^{14}$.

\section{Enfermedad de Behcet}

Enfermedad inflamatoria multisistémica crónica, y caracterizada por 3 manifestaciones recurrentes: úlceras orales, úlceras genitales y uveítis.

\section{Epidemiología}

Es más frecuente en japoneses y pobladores de las zonas orientales de la cuenca mediterránea, afecta por igual a hombres y mujeres, adultos jóvenes; se ha descrito que en hombres su evolución es más agresiva. Se ha asociado a los antígenos de histocompatibilidad HLA DR5 y B51, lo que implica una predisposición inmunogenética. Se estima una prevalencia de 0.33 casos por 100,000 personas ${ }^{4}$.

\section{Manifestaciones sistémicas}

Úlceras orales recurrentes, con 3 o más episodios anuales (primera manifestación de la enfermedad), las
Tabla 2. Criterios diagnósticos de McDonald para esclerosis múltiple progresiva primaria

1. Un año de progresión de la enfermedad (forma retrospectiva 0 a determinar prospectivamente)

2. Al menos 2 de los 3 siguientes:

A) Evidencia de diseminación de lesión en espacio, en el cerebro de al menos una lesión en T2, como mínimo en una zona característica de la EM (periventricular, yuxtacortical o infratentorial)

B) Evidencia de diseminación de lesión en espacio en la médula espinal basada en al menos 2 lesiones en T2 en la médula

C) Resultado positivo en el LCR (pruebas de isoelectroenfoque de bandas oliclonales $\mathrm{y} / 0$ índice de $\mathrm{IgG}$ elevado)

úlceras con una base necrótica amarillenta, curan en 1-2 semanas sin dejar cicatriz. Las ulceras genitales también son dolorosas, se presentan en un $80 \%$ de los casos, tienen aspecto similar a las orales, pero sí dejan cicatriz, en las mujeres la afección de la vulva puede ser subclínica, en los hombres el escroto generalmente está afectado. En piel puede observarse foliculitis $(80 \%)$, eritema nudoso más común en mujeres, afecta sobre todo piernas, puede también ser doloroso (50\%); y las erupciones acneiformes más frecuente en hombres, se encuentras sobre todo en tronco y extremidades. La artritis se observa hasta en el $60 \%$ de los pacientes, es más común en rodillas, tobillos, muñecas y codos, es asimétrica, no deformante y puede afectar una 0 varias articulaciones.

Pueden presentarse aneurismas de la arteria pulmonar siendo esta complicación fatal, generalmente afecta hombres. El involucro venoso es más común, generalmente se manifiesta como una tromboflebitis superficial aunque puede afectar venas de mediano calibre. Las manifestaciones clínicas dependen del sitio involucrado. El tracto gastrointestinal (esófago y región ileocecal) y el genitourinario (uretritis estéril, epididimitis, vejiga neurogénica) también pueden afectarse. Se ha reportado involucro renal (nefritis, glomerulonefritis) y cardiaco (vasculitis coronaria, pericarditis, miocarditis) ${ }^{15}$.

Las manifestaciones neurológicas se presentan en las fases tardías de la enfermedad, son generalmente parenquimatosas o del tallo cerebral, afectando más frecuentemente memoria o conducta. Menos comúnmente se pueden observar manifestaciones de infarto secundario a vasculitis o trombosis, meningoencefalitis o desmielinización ${ }^{16}$. 


\section{Manifestaciones oculares}

Se presenta hasta en un $70-80 \%$ de los pacientes. La afección ocular es bilateral en el $78.1 \%$ de los ca$\operatorname{sos}^{17}$. En el $10-15 \%$ se hace el diagnóstico de la enfermedad sistémica a partir de la enfermedad ocular, aunque en la mayoría de los casos las aftas orales o genitales se presentan 3-4 años antes ${ }^{18}$. Las uveítis anteriores $(48 \%)$ característicamente tienen un hipopion que cambia de posición al variar de posición del paciente $(26 \%)$, el hipopion se forma y desaparece rápidamente sin dejar secuela. Pueden presentar dolor, hiperemia conjuntival y fotofobia. En polo posterior la lesión característica es la vasculitis de retina, que afecta tanto arteria como venas. En retina se observan hemorragias, edema, exudados e infiltrados, también puede observarse papilitis. La vasculitis puede ocasionar trombosis con la consecuente isquemia retiniana, también se puede encontrar obstrucción de rama venosa retiniana $(68 \%)^{19,20}$. La panuveítis es la forma de afección más común. En casos severos puede encontrarse neovasculizarización retiniana y glaucoma neovascular.

Los episodios inflamatorios recurrentes causan finalmente atrofia de retina y de papila.

\section{Diagnóstico}

Es clínico y de acuerdo con los criterios internacionales para la enfermedad de Behcet se requieren 3 puntos (Tabla 3) 21,22.

Los diagnósticos diferenciales de enfermedad de Behcet con manifestaciones oculares y sistémicas son sífilis, lupus eritematoso sistémico, VIH y herpes en pacientes inmunodeprimidos principalmente.

\section{Tratamiento}

La finalidad del tratamiento es suprimir la inflamación, reducir la frecuencia y severidad de las recaídas y limitar la afección de retina. Debe ser agresivo cuando hay afección ocular por las complicaciones visuales graves. La liga europea contra el reumatismo (EU$L A R)$ describió nuevas guías de tratamiento. El tratamiento antiinflamatorio sistémico con corticoesteroides se puede utilizar en episodios agudos pero para uso crónico se requieren inmunosupresores sistémicos como ciclosporina. De igual manera se pueden utilizar inyecciones perioculares de esteroides ${ }^{23}$. Agentes biológicos se han usado últimamente para prevención de pérdida visual en casos severos de
Tabla 3. Criterios internacionales para la enfermedad de Behcet (positivo con 3 o más puntos)

\begin{tabular}{|c|c|}
\hline Criterios & Puntos \\
\hline Úlceras genitales recurrentes & 2 \\
\hline $\begin{array}{l}\text { Lesión ocular: uveítis anterior, uveítis posterior o } \\
\text { vasculitis de retina }\end{array}$ & 2 \\
\hline Úlceras orales recurrentes & 1 \\
\hline Lesiones en piel: eritema nudoso o seudofoliculitis & 1 \\
\hline $\begin{array}{l}\text { Manifestaciones vasculares: flebitis superficial, } \\
\text { trombosis venosa profunda, trombosis de grandes } \\
\text { vasos, trombosis arterial y aneurismas }\end{array}$ & 1 \\
\hline Test de patergia positivo (Behcetine test) ${ }^{*}$ & 1 \\
\hline \multicolumn{2}{|c|}{$\begin{array}{l}\text { *Leído a las } 24-48 \text { h. Consiste en causar microtraumatismo con aguja estéril en la } \\
\text { epidermis de la superficie cutánea interna del antebrazo y en la cual se espera } \\
\text { que se forme una pápula o pústula. Es causada por una hiperreactividad } \\
\text { inflamatoria o hiperreactividad inespecífica de la piel producida por una } \\
\text { acumulación de polimorfonucleares en las capas más superficiales de la epidermi } \\
\text { con producción anormal de interleucina } 2 \text {. }\end{array}$} \\
\hline
\end{tabular}

uveítis por Behcet, sin embargo no hay estudios clínicos controlados hasta el momento. Se han visto buenos resultados con anticuerpos monoclonales anti-TNF alfa, infliximab (5 mg/kg en infusión) en pacientes con uveítis por Behcet resistente a tratamiento convencional, ataques graves o con riesgo alto de pérdida visual. El interferón alfa es efectivo en el $90 \%$ de los $\operatorname{casos}^{24}$.

\section{Pronóstico}

El pronóstico de perdida visual $(>0.1)$ de 5 a 10 años es del 21 al $10 \%$ en hombres y del 30 al $17 \%$ en mujeres ${ }^{18}$. Los hombres jóvenes tienen peor pronóstico visual. La pérdida visual de 20/200 o peor es de 0.09 por año ${ }^{24}$.

\section{Sarcoidosis}

Enfermedad inflamatoria multisistémica de etiología desconocida que se manifiesta con granulomas no caseificantes, predominantemente en pulmones y en nódulos linfoides.

\section{Epidemiología}

Afecta a cualquier edad, pero principalmente a pacientes de 20-39 años. La incidencia más alta es en Europa, con 5-40 casos por 100,000 habitantes ${ }^{25}$. La vasculitis sistémica en sarcoidosis puede ser responsable del $1.4 \%$ de los casos de vasculitis retiniana ${ }^{26}$. 
Tabla 4. Diagnóstico de sarcoidosis ocular según el "International Workshop On Ocular Sarcoidosis»

\begin{tabular}{|l|l|}
\hline Diagnóstico definitivo de sarcoidosis ocular & Diagnóstico corroborado por biopsia y uveítis compatible \\
\hline Presunta sarcoidosis ocular & $\begin{array}{l}\text { Si la biopsia no es realizada pero la radiografía de tórax es positiva, mostrando } \\
\text { linfadenopatía hiliar bilateral, asociada a uveítis compatible }\end{array}$ \\
\hline Probable sarcoidosis ocular & $\begin{array}{l}\text { Si la biopsia no fue realizada y la radiografía de tórax no muestra linfadenopatía hiliar } \\
\text { bilateral, pero hay } 3 \text { signos intraoculares y } 2 \text { pruebas de laboratorio positivas }\end{array}$ \\
\hline Posible sarcoidosis ocular & $\begin{array}{l}\text { Si la biopsia pulmonar fue realizada y el resultado es negativo, pero al menos } 4 \text { signos } \\
\text { intraoculares y } 2 \text { pruebas de laboratorio positivas estuvieron presentes }\end{array}$
\end{tabular}

\section{Manifestaciones sistémicas}

Puede cursar asintomática en un $5 \%$ de los casos, las manifestaciones pulmonares (disnea de esfuerzo, tos y dolor de pecho) se presentan en el $50 \%$ de los casos. El síndrome de Löfgren (poliartralgias, adenopatía hiliar bilateral y fiebre) se observa más comúnmente en pacientes escandinavos que en afroamericanos o japoneses. Las manifestaciones dermatológicas más frecuentes son eritema nudoso, lupus pernio, rash violáceo en mejillas y nariz. El involucro cardiaco está caracterizado por taquiarritmias ventriculares en el $15 \%$ de los pacientes. Con menor frecuencia se ha reportado involucro óseo, cardiovascular, meningitis linfocítica y parálisis de nervios craneales ${ }^{25}$.

\section{Manifestaciones oculares}

El involucro ocular puede presentarse hasta en el $60 \%$ de los pacientes con sarcoidosis sistémica ${ }^{27}$. La afección de la glándula lagrimal se encuentra en una tercera parte de los pacientes, manifestándose como edema palpable no doloroso, que puede causar queratitis sicca. Si se afecta la cavidad orbitaria la manifestación más común es la proptosis.

La uveítis anterior granulomatosa bilateral es la presentación más común. En el $25-30 \%$ de los pacientes se observan manifestaciones en polo posterior como opacidades vítreas blanquecinas («bolas de nieve» 0 "collares de perlas»), edema macular, granulomas coroideos, granuloma de nervio óptico (Fig. 2) y neovascularizacion retiniana. La vasculitis de retina en la forma de periflebitis es característica de la enfermedad (8.7\%), en algunos casos tiene aspecto de "gota de cera", pero lo común es que solo se vea envainamiento ${ }^{26}$. En el $38 \%$ de los casos de sarcoidosis ocular se puede diagnosticar sarcoidosis sistémica, principalmente en mujeres mayores de 60 años con uveítis crónicas. La bilateralidad de la enfermedad ocular se ha reportado hasta en el $84 \%$ de los casos cuando hay panuveitis ${ }^{28}$ (Fig. 2).
Tabla 5. Signos de sarcoidosis ocular

\section{Precipitados retroqueratósicos pequeños o en grasa de} carnero, iris con nódulos de Koeppe/Busacca

Nódulos en malla trabecular o sinequias anteriores en tienda de campaña

Opacidades vítreas en forma de copos de nieve/collar de perlas

Lesiones múltiples coriorretinianas periféricas (activa 0 atrófica)

Periflebitis nodular o segmentaria, en forma de "gota de cera" y/o macroaneurismas retinianos en ojos inflamados

Nódulos en disco óptico, granuloma o nódulo coroideo solitario Bilateralidad

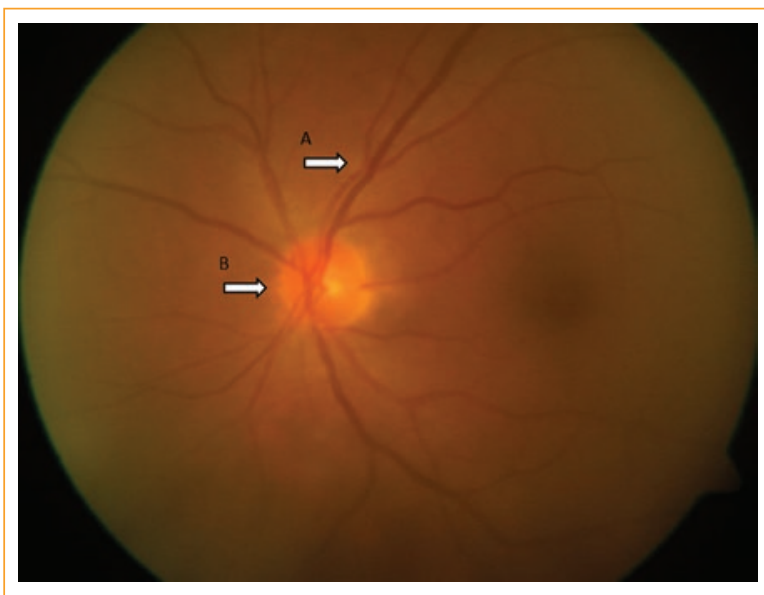

Figura 2. Nódulo coroideo (A) y nódulo de nervio óptico (B) en sarcoidosis ocular.

\section{Diagnóstico}

Actualmente existen criterios precisos de sarcoidosis ocular resultado del primer «International Workshop On Ocular Sarcoiodosis» 29 (Tablas 4 y 5). 
Tabla 6. Estadios radiológicos de sarcoidosis pulmonar

Estadio 0: ausencia de alteraciones

Estadio 1: hay adenopatías hiliares bilaterales y pueden verse otras adenopatías acompañantes, fundamentalmente paratraqueales derechas y en la ventana aortopulmonar

Estadio 2: se observan adenopatías hilares bilaterales y afectación parenquimatosa, con patrón micronodular o reticulonodular bilateral, simétrico, de predominio perihiliar, en los campos pulmonares medios y superiores

Estadio 3: hay afectación parenquimatosa sin adenopatías hiliares

Estadio 4: se observa fibrosis pulmonar, manifestada como patrón reticular con bronquiectasias de tracción, masas con distorsión de la arquitectura pulmonar o quistes de panal, con predominio en los campos superiores

Tabla 7. Hallazgos típicos de sarcoidosis en tomografía axial computarizada de tórax

Adenopatías: hiliares simétricas y paratraqueales derechas; además, mediastínicas medias y anteriores

Patrón nodulillar linfangítico:

Tipo nódulos: < de $10 \mathrm{~mm}$, más frecuentes entre 2 y $5 \mathrm{~mm}$ bien definidos, lisos 0 irregulares

Tipo de distribución: linfangítica, parcheada, predominio en pulmón central o periférico (más frecuente) y predominio en campos superiores y medios

Signos "del corro de brujas" y "del cúmulo sarcoideo»*

Afectación bronquiolar: atrapamiento aéreo en espiración ${ }^{\dagger}$

Fibrosis: bronquiectasias de tracción en LLSS, panal en los campos medios

* Descritos en sarcoidosis. Solo un caso de cúmulo sarcoideo descrito en tuberculosis.

†Hallazgo no específico, aunque el segundo en frecuencia después del patrón nodulillar linfangítico.

Existen estudios con valor diagnóstico en asociación con signos de sarcoidosis ocular que son los siguientes:

- Prueba de tuberculina negativa en un paciente previamente vacunado o con prueba positiva previa.

- Niveles elevados de enzima convertidora de angiotensina o lisozima sérica.

- Radiografía de tórax con infiltrados hiliares bilaterales.

- Alteración de enzimas hepáticas.

- Tomografía axial computarizada de tórax positiva en un paciente con radiografía negativa.

En pacientes que no presenten granulomas no caseificantes para biopsiar o en los que la radiografía de tórax sea negativa (Tabla 6) y se sospeche de sarcoidosis se puede realizar tomografía computarizada de tórax (Tabla 7$)^{30}$.

\section{Tratamiento}

Los corticoesteroides son la base del tratamiento; con la administración de prednisona vía oral $1 \mathrm{mg} / \mathrm{kg} / \mathrm{día}$. Cuando existe involucro ocular se pueden utilizar esteroides perioculares. Se puede utilizar inmunosupresores como metotrexate, ciclofosfamida y azatioprina.
Estudios recientes usan terapia antimicobacteriana (levofloxacino, etambutol, azitromicina, rifampicina) para el tratamiento de sarcoidosis ocular y cutánea con buenos resultados ${ }^{31}$.

\section{Pronóstico}

Cuando hay alteración ocular, después de 10 años, el $54 \%$ de los pacientes mantienen su agudeza visual y el $4.6 \%$ tienen pérdida de visión peor que $20 / 100$ en ambos ojos. El $51 \%$ requieren esteroides orales para el tratamiento de uveítis y el $11 \%$ el uso de inmunosupresores. Al momento de la afectación ocular el 37\% de los casos se ha demostrado la afección pulmonar ${ }^{32}$. Las complicaciones oculares comunes son sinequias posteriores, catarata y glaucoma, otras complicaciones son cambios en polo posterior y neovascularizaciones coroideas peripapilares y subfoveales. En enfermedad sistémica, un $30-50 \%$ de los casos mejoran sin tratamiento en un periodo de 3 años, alrededor del $20 \%$ de las personas con alteración pulmonar tendrán daño pulmonar permanente. La tasa de moralidad total por sarcoidosis es del $5 \%$, las causas de muerte son hemorragia pulmonar, insuficiencia cardiaca y fibrosis pulmonar ${ }^{33}$. 


\section{Financiamiento}

Los autores no recibieron patrocinio para llevar a cabo este artículo.

\section{Conflicto de intereses}

\section{Los autores declaran no tener ningún conflicto de intereses.}

\section{Bibliografía}

1. Neal V. Ocular manifestations of systemic lupus erythematosus: A review of the literature. Autoinmune Dis. 2012:1-9.

2. Gonzalez F. Criterios diagnósticos y de clasificación de las enfermedades reumáticas. Guías de práctica clínica Sociedad Española de Reumatología. 2011 [consultado 28Sep 2015]. Disponible en: http://www.ser.es/ practicaClinica/Criterios Diagnosticos.php.

3. Muscal E, Brey R. Neurological manifestations of systemic lupus erythematosus in children and adults. Neurol Clin.2010;28:61-73.

4. Graham EM, Stanford MR, Sanders MD, Kasp E, Dumonde DC. A point prevalence study of 150 patients with idiopathic retinal vasculitis: 1 . Diagnostic value of ophthalmological features. $\mathrm{Br} \mathrm{J}$ Ophthalmol. 1989;73:714-21.

5. Au A, O'Day J. Review of severe vaso-occlusive retinopathy in systemic lupus erythematosus and the antiphospholipid syndrome: Associations, visual outcomes, complications and treatment. Clin Experiment Ophthalmol. 2004;32:87-100.

6. Yazici A. The biomechanical properties of the cornea in patients with systemic lupus erythematosus. Eye Lond. 2011;25:1005-9.

7. Hernández Jl, Castañeda G, Rodríguez de Romo AC. Esclerosis múltiple en México: la clínica de su historia. Rev Fac Med UNAM. 2006:49:73-8.

8. Gordon LK. Gender and uveitis in patients with multiple sclerosis. J Ophamol. 2014:1-5

9. Perez R, Gomez A. Optic neuritis in pediatric population: A review in current tendencies of diagnosis and mangement. J Optom. 2014;7:125-30.

10. Chen LG. Ocular manifestations of multiple sclerosis. Curr Opin Ophthalmol. 2005;16:315-20.

11. Rio J. Retinal periphlebit is in multiple sclerosis. A prospective study. Neurol J. 1993;8:252-5
12. Ahmed M. Differential diagnosis of retinal vasculitis. Middle East Afr $J$ Ophthalmol. 2009;16:202-18.

13. Polman $\mathrm{CH}$. Diagnostic criteria for multiple sclerosis 2010 revisions to the McDonald criteria. Ann Neurol. 2011;69:292-302.

14. Mackensen F. Interferon therapy for ocular disease. Curr Opin Ophtalmol. 2006;17:567-73.

15. Davatchi $F$, Chams D. Adult Behcet's disease in Iran: Analysis of 6075 patients. Int J Rheum Dis. 2015;10:117.

16. Al-Araji A, Kidd D. Neuro-Behçet's disease: Epidemiology, clinical characteristics, and management. Lancet Neurol. 2009;8:192-204.

17. Michelson JB, Chisari FU. Behcet's disease. Surv Ophthalmol.1982:26: 190-203.

18. Tugal T. Uveitis in Behcet disease: An analysis of 880 patients. Am J Ophthalmol. 2004;138:373-80.

19. Al-Dhibi H. Macular hole in Behcet's disease. Indian J Ophtalmol. 2011;59:359-62

20. Mujaini A. Frosted branch angiitis, neuroretinitis as initialocular manifestation in Behcet disease. Indian J Ophthalmol. 2011:59:240-1.

21. Fereydoun D. Diagnosis/Classification criteria for Behcet's disease. Patholog Res Int. 2012:1-5.

22. Pimienta M. El fenómeno de patergia. Semin Fund Esp Reumatol. 2009:10. Disponible en: http://www elsevier.es/es-revista-seminarios-fundacion-espanola-reumatologia-274-articulo-el-fenomeno-patergia-13140685 [consultado 5 Mar 2015].

23. Hatemi GS. EULAR recommendations for the management of Behcet disease. Ann Rheum Dis. Dec. 2008;67:1656-62.

24. Tugal-Tutkun I. Behcet's uveitis. Middle East Afr J Ophthalmol. 2009; 16:219-24

25. lannuzzi MC Sarcoidosis. N Engl J Med. 2007;357:2153-65.

26. James $T$. Patients with retinal vasculitis rarely suffer from systemic vasculitis. Semin Arthritis Rheum. 2012;41:859-65.

27. Takase $\mathrm{H}$. Validation of international criteria for the diagnosis of ocular sarcoidosis proposed by the first international workshop on ocular sarcoidosis. Jpn J Ophthalmol. 2010;54:529-36.

28. Adan A. Uveitis como manifestación inicial de sarcoidosis. Med Clin (Barc). 2004;122(19):748-52.

29. Herbort CP, Rao NA. International criteria for the diagnosis of ocular sarcoidosis: Results of the first International Workshop On Ocular Sarcoidosis (IWOS). Ocul Immunol Inflamm. 2009;17:160-9.

30. Herráez OA. La sarcoidosis torácica. Radiología. 2011;53:434-48.

31. Bradley W. Resolution of chronic ocular sarcoidosis with antimycobacterial therapy. Case Rep Intern Med. 2014:1:5042.

32. Edelsten $\mathrm{C}$. The ocular and systemic prognosis of patients presenting with sarcoid uveitis. Eye Lond. 1999;13:748-53.

33. Drake W, Newman L. Sarcoidosis. En: Mason RJ, Broaddus VC Martin Tr, et al, editores. Murray and Nadel's Textbook of Respiratory Medicine. $5^{\text {th }}$ ed. Philadelphia, PA: Elsevier Saunders; 2010. p. 59. 